\title{
Descending endografts for type A dissections: con
}

\author{
Akiko Tanaka, Harleen K. Sandhu, Anthony L. Estrera \\ Department of Cardiothoracic and Vascular Surgery, McGovern Medical School at The University of Texas Health Science Center at Houston \\ (UTHealth) and Memorial Hermann Hospital, Houston, TX, USA \\ Correspondence to: Anthony L. Estrera, MD, FACS. Department of Cardiothoracic and Vascular Surgery, McGovern Medical School at UTHealth, \\ 6400 Fannin St, Ste. 2850, Houston, TX 77030, USA. Email: Anthony.L.Estrera@uth.tmc.edu.
}

\begin{abstract}
An important goal of surgical repair of type A aortic dissection is to resect the intimal tear. Studies on the fate of residual dissection after acute type A aortic dissection repair in the past decade have driven surgeons to seek procedures to avoid distal reoperation. Aggressive surgical approach with total arch replacement in acute type A dissection has demonstrated lower incidence of distal reoperation and slower aortic growth rate compared to less aggressive ascending/hemiarch repair. Recently, successful results of thoracic endovascular aortic repair (TEVAR) in type B aortic dissection have encouraged surgeons to further attack acute type A aortic dissection with hybrid approach: antegrade endografting in the descending thoracic true lumen as an adjunct to hemiarch or total arch repair. However, is the hybrid approach with simultaneous descending endografting justified in all the acute type A aortic dissection cases? The outcomes of the hybrid approach reported by the several groups have demonstrated $80-100 \%$ of false lumen thrombus formation in the proximal descending aorta (stented segment); however, the complete obliteration of the false lumen is only $17-50 \%$. The incidence of distal reoperation/reintervention after hybrid approach is as high as $14 \%$ and an adjunctive procedure is often performed. Moreover, there are concerns about additional risks associated with the hybrid procedures, such as spinal cord injury (SCI), stent graft induced new entry and stroke. The data on this new approach are still very limited. Hence, further study is warranted to prove its safety and durability.
\end{abstract}

Keywords: Acute type A aortic dissection; endograft; hybrid

Submitted Feb 29, 2016. Accepted for publication Apr 08, 2016.

doi: $10.21037 /$ acs.2016.04.03

View this article at: http://dx.doi.org/10.21037/acs.2016.04.03

\section{Introduction}

Recent publication based on the 17-year trends from the International Registry of Acute Aortic Dissection showed that in-hospital mortality after surgical repair of acute type A aortic dissection has improved from $25 \%$ to $18 \%$ (1). Due to increased experience with this lethal disease (2-16), reports on the fate of residual dissection have been published after the late 2000s (Table 1). Risk factors for distal reoperation have been identified as non-resected intimal tear, Marfan's syndrome, younger age, high blood pressure during follow up, large aortic size at the initial presentation and large false lumen. Although an important goal of surgical repair of type A aortic dissection is to resect the intimal tear, there is a long-standing debate on whether to perform hemiarch replacement or total arch replacement in patients with younger age, connective tissue disorder, large aortic arch or disrupted arch $(15,16)$ when the tear is located in the ascending aorta. An aggressive approach with total arch replacement has demonstrated lower incidence of aortic events, including distal reoperation or aortic dilation compared to conservative hemiarch repair $(2,3,5,16)$. The incidence of distal reoperation after type A repair with ascending/hemiarch replacement in contemporary series varies from $8.3-15.7 \%(4,6,9,13,16)$, while that of total arch replacement is $3.8-4.3 \%(5,16)$. In addition, early mortality rate after total arch replacement in acute type A aortic dissection is reported as $3.7-20.4 \%(2,3,5,15,17)$, which is comparable to results with less aggressive procedures. These results have led to aggressive total arch replacement 


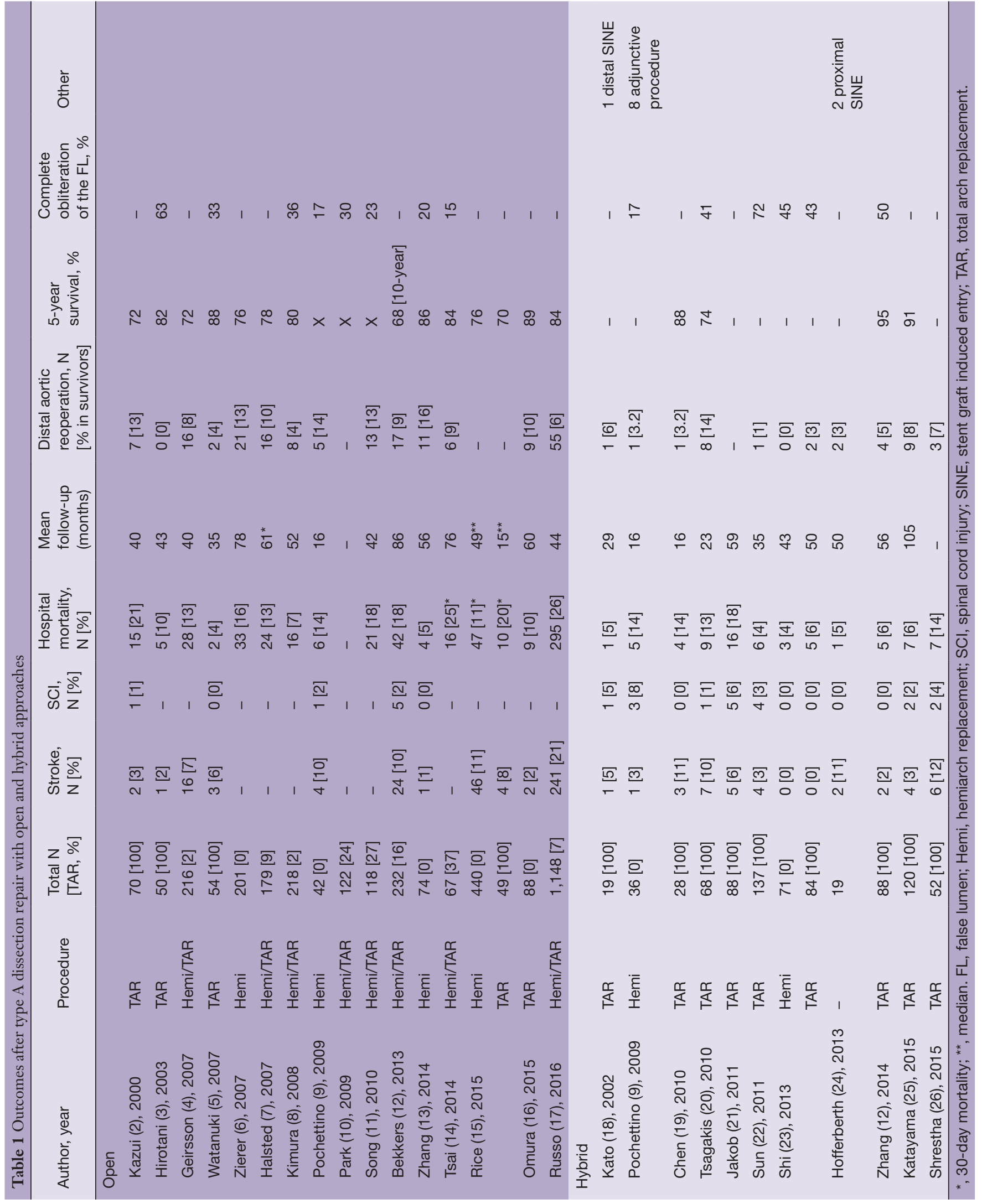


in type A dissection to be accepted as a surgical option in the selected patient population mentioned above.

\section{Hybrid approach in acute type A aortic dissection}

\section{Background}

Numbers of reports on treating acute type A aortic dissection with a hybrid approach, open ascending/hemiarch or total arch repair with antegrade descending endografting have dramatically increased in the past decade $(9,12,18-26)$. The antegrade or retrograde endografting of the descending aorta is often referred as 'frozen elephant trunk', 'stented elephant trunk' or 'adjunct thoracic endovascular aortic repair (TEVAR)'. Successful outcomes of TEVAR in type $\mathrm{B}$ aortic dissection (27-30) have accelerated the application of hybrid approach to treat acute type A dissection. Randomized trials on type B aortic dissection with TEVAR demonstrated consistent positive false lumen remodeling in the descending aorta leading to low aortic events. Thus, it is not surprising that surgeons are now trying to treat type A dissection with the hybrid approach.

Malperfusion syndrome is observed in $20-40 \%$ of patients with acute type A aortic dissection. Malperfusion may resolve spontaneously after a central repair, but nearly $20 \%$ require additional revascularization (31). Thus, simultaneous endografting in the descending aorta in the complicated malperfusion cases may be a good treatment option, especially when the malperfusion is caused by the narrowing of the true lumen. However, the question remains: Is the hybrid approach to treat acute type A dissection justified in all the cases? There are always concerns about additional complications associated with an additional procedure. The hybrid approach may cause complications, such as stent graft-induced new entry (SINE) tear (32), spinal cord injury (SCI) (33) and stroke.

\section{Sizing technique}

Sizing technique of the hybrid repair in type A dissection differs from institution to institution and is not standardized. The reported sizing techniques are: size of the true lumen as measured by intraoperative sizing with a ball sizer (12,25), 10-15\% larger than the proximal aorta measured by preoperative computed tomography (CT) (9), size only of the true lumen, obtained by preoperative CT (20), size of total lumen diameter obtained by preoperative CT (24), or intraoperative angiogram (22). Sizing of the endograft for the hybrid approach is crucial because the endograft needs to be large enough to open up the collapsed true lumen and avoid type I endoleak. But, in the meantime, oversizing would cause SINE (33). SINE can cause catastrophic complication after hybrid type A dissection repair $(17,24)$. We have recently experienced a difficult reoperation case that was treated with hemiarch repair and descending endografting of acute type A aortic dissection. The patient had SINE in the distal arch proximal to the stent and required urgent reoperation due to rapid expanding arch aneurysm. We believe this complication was due to oversizing of the endograft. Thus, further studies to establish the sizing technique of the endograft are needed before this approach becomes available to non-experienced hands.

\section{Spinal cord injury (SCI)}

The incidence of SCI after the hybrid technique of arch repair and open antegrade endografting in the descending aorta for acute type A aortic dissection is $0-8 \%(9,12,18-26)$. The mechanism of increased incidence of SCI in hybrid technique compared to isolated TEVAR in the type $\mathrm{B}$ dissection may be explained as a lower distal perfusion pressure due to the open distal technique (33). Cerebral spinal drainage, perfusion of left subclavian artery and distal perfusion with balloon occlusion technique are utilized by some groups to avoid SCI in hybrid approach (22-24). In fear of SCI, the commonly used length of endograft is around $10-15 \mathrm{~cm}(9,19-21,25)$, which is shorter than the average length of endografts used in type B dissection. This shorter endograft may be controversial in terms of positive aortic remodeling. As demonstrated in the outcomes after treating type B aortic dissection with TEVAR, false lumen thrombosis after the hybrid approach in type A dissection is often not obtained beyond the stent graft segment because there the secondary tear often exists between the true and false lumens (33).

\section{Stroke}

Reported stroke rates of $0-12 \%(9,12,18-26)$ after hybrid procedure treating type A aortic dissection does not seem inferior to conventional hemiarch or total arch replacement. However, we should be aware that the numbers of reported hybrid cases are very limited and patient selection is often biased (e.g., unstable hemodynamics with a stroke 
and pulmonary deficiency are not indicated for hybrid approach) (25). Moreover, most centers have added selective antegrade cerebral perfusion $(9,12,18-26)$ to their procedure to avoid stroke due to the longer open distal time compared to the conventional arch repair. Thus, hybrid procedures may be performed with acceptable risk of stroke when proper cerebral protection is provided in a selected patient group.

\section{Distal reoperation}

It has been widely reported that complete false lumen thrombosis results in better outcomes after type A aortic repair and partial thrombosis of the false lumen is identified as a risk factor of late aortic events. Although the false lumen thrombosis is observed in $80-100 \%$ in the stented segment of the proximal descending aorta $(18,21,22,25)$, complete false lumen thrombosis rate after hybrid type A dissection repair is limited to $17-50 \%(9,12,20,23)$, which is similar to that of total arch replacement with elephant trunk (43-63\%) (3,5). Despite the fact that the history of the hybrid approach in the type A dissection is relatively short, the reported distal reoperation rate is $0-14 \%$ $(9,12,18-20,22-26)$ and has no superiority to total arch replacement with elephant trunk $(0-13 \%)(2,3,5,16)$. In addition to the distal reinterventions, adjunctive procedures such as branch vessel intervention or additional stenting to cover endoleak or SINE, are often performed after the hybrid approach.

Distal reoperation for chronic descending aortic dissection can be performed with respectable morbidity and mortality in general $(34,35)$ However, it is often technically challenging to perform open surgical repair in patients who had previous endograft repair in the descending aorta due to intimal damage and adhesion to surrounding tissue caused by the endograft. Szeto et al. (35) reported high postoperative dialysis rate (15\%) and mortality $(15 \%)$ in a series of open reinterventions for TEVAR failure.

\section{Value}

Cost effectiveness and value research of many operative procedures is seldom compared, let alone reported. No data regarding this aspect exists in relation to antegrade stenting for acute type A aortic dissection. As prior studies have not demonstrated a reduction in hospital length of stay with the hybrid procedures, it stands to reason that the procedural costs of the antegrade stent grafting would be greater than the standard graft replacement, thus reducing its valve.

\section{Survival}

Lastly, would the hybrid procedure improve survival of acute type A dissection? In-hospital mortality after hybrid therapy for type A aortic dissection is documented as $4.2-14.3 \%(9,12,18-20,22-26)$, which is comparable to conventional open repair. Moreover, there is not enough data demonstrating survival benefits of the hybrid therapy in mid-term. The failure of proving superiority of hybrid therapy to conventional approach in type A dissection may be extracted from what was observed in the studies of TEVAR in acute type B dissection. Close observation alone may have provided improved survival rates in type A dissection patients. Accumulation of long-term data is necessary to clarify the survival benefit of endografting in the descending aorta in type A aortic dissection in the future.

\section{Summary}

Hybrid approach of open hemiarch/arch repair and endografting of the descending aorta in acute type A aortic dissection may be a good option in patients with distal malperfusion with collapsed true lumen and large descending aorta when it can be performed in experienced hands. With the evolution of stent graft product and further accumulation of data, especially sizing and length of the stent grafts, this technique may become feasible for extended indications. However, further investigation on safety and long-term durability is warranted to conclude the true benefit and proper application of hybrid procedures in acute type A aortic repair.

\section{Acknowledgements}

The authors thank Troy D. Brown for editorial assistance.

\section{Footnote}

Conflicts of Interest: Anthony L. Estrera is a consultant for Gore. The other authors have no conflicts of interest to declare.

\section{References}

1. Pape LA, Awais M, Woznicki EM, et al. Presentation, 
Diagnosis, and Outcomes of Acute Aortic Dissection: 17Year Trends From the International Registry of Acute Aortic Dissection. J Am Coll Cardiol 2015;66:350-8.

2. Kazui T, Washiyama N, Muhammad BA, et al. Extended total arch replacement for acute type a aortic dissection: experience with seventy patients. J Thorac Cardiovasc Surg 2000;119:558-65.

3. Hirotani T, Nakamichi T, Munakata M, et al. Routine extended graft replacement for an acute type A aortic dissection and the patency of the residual false channel. Ann Thorac Surg 2003;76:1957-61.

4. Geirsson A, Bavaria JE, Swarr D, et al. Fate of the residual distal and proximal aorta after acute type a dissection repair using a contemporary surgical reconstruction algorithm. Ann Thorac Surg 2007;84:1955-64; discussion 1955-64.

5. Watanuki H, Ogino H, Minatoya K, et al. Is emergency total arch replacement with a modified elephant trunk technique justified for acute type A aortic dissection? Ann Thorac Surg 2007;84:1585-91.

6. Zierer A, Voeller RK, Hill KE, et al. Aortic enlargement and late reoperation after repair of acute type A aortic dissection. Ann Thorac Surg 2007;84:479-86; discussion 486-7.

7. Halstead JC, Meier M, Etz C, et al. The fate of the distal aorta after repair of acute type A aortic dissection. J Thorac Cardiovasc Surg 2007;133:127-35.

8. Kimura N, Tanaka M, Kawahito K, et al. Influence of patent false lumen on long-term outcome after surgery for acute type A aortic dissection. J Thorac Cardiovasc Surg 2008;136:1160-6, 1166.e1-3.

9. Pochettino A, Brinkman WT, Moeller P, et al. Antegrade thoracic stent grafting during repair of acute DeBakey I dissection prevents development of thoracoabdominal aortic aneurysms. Ann Thorac Surg 2009;88:482-9; discussion 489-90.

10. Park KH, Lim C, Choi JH, et al. Midterm change of descending aortic false lumen after repair of acute type I dissection. Ann Thorac Surg 2009;87:103-8.

11. Song SW, Chang BC, Cho BK, et al. Effects of partial thrombosis on distal aorta after repair of acute DeBakey type I aortic dissection. J Thorac Cardiovasc Surg 2010;139:841-7.e1; discussion 847.

12. Bekkers JA, Raap GB, Takkenberg JJ, et al. Acute type A aortic dissection: long-term results and reoperations. Eur J Cardiothorac Surg 2013;43:389-96.

13. Zhang H, Lang X, Lu F, et al. Acute type A dissection without intimal tear in arch: proximal or extensive repair?
J Thorac Cardiovasc Surg 2014;147:1251-5.

14. Tsai MT, Wu HY, Roan JN, et al. Effect of false lumen partial thrombosis on repaired acute type A aortic dissection. J Thorac Cardiovasc Surg 2014;148:21402146.e3.

15. Rice RD, Sandhu HK, Leake SS, et al. Is Total Arch Replacement Associated With Worse Outcomes During Repair of Acute Type A Aortic Dissection? Ann Thorac Surg 2015;100:2159-65; discussion 2165-6.

16. Omura A, Miyahara S, Yamanaka K, et al. Early and late outcomes of repaired acute DeBakey type I aortic dissection after graft replacement. J Thorac Cardiovasc Surg 2016;151:341-8.

17. Russo CF, Mariscalco G, Colli A, et al. Italian multicentre study on type A acute aortic dissection: a 33-year followup†. Eur J Cardiothorac Surg 2016;49:125-31.

18. Kato M, Kuratani T, Kaneko M, et al. The results of total arch graft implantation with open stent-graft placement for type A aortic dissection. J Thorac Cardiovasc Surg 2002;124:531-40.

19. Chen X, Huang F, Xu M, et al. The stented elephant trunk procedure combined total arch replacement for Debakey I aortic dissection: operative result and follow-up. Interact Cardiovasc Thorac Surg 2010;11:594-8.

20. Tsagakis K, Pacini D, Di Bartolomeo R, et al. Multicenter early experience with extended aortic repair in acute aortic dissection: is simultaneous descending stent grafting justified? J Thorac Cardiovasc Surg 2010;140:S116-20; discussion S142-S146.

21. Jakob H, Tsagakis K, Pacini D, et al. The International E-vita Open Registry: data sets of 274 patients. J Cardiovasc Surg (Torino) 2011;52:717-23.

22. Sun L, Qi R, Zhu J, et al. Repair of acute type A dissection: our experiences and results. Ann Thorac Surg 2011;91:1147-52.

23. Shi E, Gu T, Yu Y, et al. Simplified total arch repair with a stented graft for acute DeBakey type I dissection. J Thorac Cardiovasc Surg 2014;148:2147-54.

24. Hofferberth SC, Newcomb AE, Yii MY, et al. Hybrid proximal surgery plus adjunctive retrograde endovascular repair in acute DeBakey type I dissection: superior outcomes to conventional surgical repair. J Thorac Cardiovasc Surg 2013;145:349-54; discussion 354-5.

25. Katayama A, Uchida N, Katayama K, et al. The frozen elephant trunk technique for acute type A aortic dissection: results from 15 years of experience†. Eur J Cardiothorac Surg 2015;47:355-60; discussion 360.

26. Shrestha M, Beckmann E, Krueger H, et al. The elephant 
trunk is freezing: The Hannover experience. J Thorac Cardiovasc Surg 2015;149:1286-93.

27. Fattori R, Cao P, De Rango P, et al. Interdisciplinary expert consensus document on management of type $\mathrm{B}$ aortic dissection. J Am Coll Cardiol 2013;61:1661-78.

28. Nienaber CA, Rousseau H, Eggebrecht H, et al. Randomized comparison of strategies for type B aortic dissection: the INvestigation of STEnt Grafts in Aortic Dissection (INSTEAD) trial. Circulation 2009;120:2519-28.

29. Nienaber CA, Kische S, Rousseau H, et al. Endovascular repair of type $\mathrm{B}$ aortic dissection: long-term results of the randomized investigation of stent grafts in aortic dissection trial. Circ Cardiovasc Interv 2013;6:407-16.

30. Brunkwall J, Kasprzak P, Verhoeven E, et al. Endovascular repair of acute uncomplicated aortic type B dissection promotes aortic remodelling: 1 year results of the

Cite this article as: Tanaka A, Sandhu HK, Estrera AL. Descending endografts for type A dissections: con. Ann Cardiothorac Surg 2016;5(3):227-232. doi: 10.21037/ acs.2016.04.03
ADSORB trial. Eur J Vasc Endovasc Surg 2014;48:285-91.

31. Charlton-Ouw KM, Sritharan K, Leake SS, et al. Management of limb ischemia in acute proximal aortic dissection. J Vasc Surg 2013;57:1023-9.

32. Dong Z, Fu W, Wang Y, et al. Stent graft-induced new entry after endovascular repair for Stanford type B aortic dissection. J Vasc Surg 2010;52:1450-7.

33. Katayama K, Uchida N, Katayama A, et al. Multiple factors predict the risk of spinal cord injury after the frozen elephant trunk technique for extended thoracic aortic disease. Eur J Cardiothorac Surg 2015;47:616-20.

34. Estrera AL, Jan A, Sandhu H, et al. Outcomes of open repair for chronic descending thoracic aortic dissection. Ann Thorac Surg 2015;99:786-93; discussion 794.

35. Szeto WY, Desai ND, Moeller P, et al. Reintervention for endograft failures after thoracic endovascular aortic repair. J Thorac Cardiovasc Surg 2013;145:S165-70. 